\title{
Differences in Trauma-Related Guilt in Females with History of Sexual Violence Based on Insomnia Severity
}

\author{
Sora Han, $\mathrm{BA}^{1}$, Young Ki Chung, $\mathrm{MD}^{2,3}$, Mi Ran Kim, MD, $\mathrm{PhD}^{4}$, Hanbyul Lee, $\mathrm{MA}^{3}$, \\ Yu Jin Kim, BA ${ }^{3}$, Hyoung Yoon Chang, MD, PhD, MPH ${ }^{2,3}$, Sooyeon Suh, $\mathrm{PhD}^{1 *}$ \\ 'Department of Psychology, Sungshin Women's University, Seoul, Korea \\ ${ }^{2}$ Departments of Psychiatry, ${ }^{4}$ Obstetrics and Gynecology, Ajou University School of Medicine, Suwon, Korea \\ ${ }^{3}$ Sunflower Center of Southern Gyeonggi for Women and Children Victims of Violence, Suwon, Korea
}

\author{
Received: May 29, 2018 \\ Accepted: June 20, 2018 \\ Correspondence \\ Sooyeon Suh, PhD \\ Department of Psychology, \\ Sungshin Women's University, \\ 2 Bomun-ro 34-gil, Seongbuk-gu, \\ Seoul 02844, Korea \\ Tel +82-2-920-7215 \\ Fax +82-2-920-2040 \\ E-mail alysuh@sungshin.ac.kr \\ Hyoung Yoon Chang, MD, PhD, MPH \\ Department of Psychiatry, \\ Ajou University School of Medicine, \\ 164 World cup-ro, Yeongtong-gu, \\ Suwon 16499, Korea \\ Tel +82-31-217-9117 \\ Fax +82-31-217-5198 \\ E-mail hyoungyoon@ajou.ac.kr
}

*These authors contributed equally to this work.

ORCID

Sora Han

https://orcid.org/0000-0001-9431-1034 Young Ki Chung

https://orcid.org/0000-0002-7822-6152 Mi Ran Kim

https://orcid.org/0000-0001-5553-5334 Hanbyul Lee

https://orcid.org/0000-0003-0903-3952

Yu Jin Kim

https://orcid.org/0000-0003-3624-0092

Hyoung Yoon Chang

https://orcid.org/0000-0002-5248-3433

Sooyeon Suh

https://orcid.org/0000-0003-0644-8634
Background and Objective Females with history of sexual violence report a high percentage of insomnia. Guilt is a common symptom among this group. This study investigated differences in trauma-related guilt cognition between females of sexual violence with high or low symptoms of insomnia.

Methods Participants were 43 females who reported having a history of sexual violence (mean age 26.56 \pm 7.81 ). All participants completed questionnaires about insomnia symptoms (Insomnia Severity Index, ISI), Posttraumatic stress disorder (PTSD) symptoms (PTSD Symptom Scale SelfReport), trauma-related guilt (Trauma-related Guilt Inventory, TRGI), depression (Beck Depression Inventory) and trauma-related information. The TRGI is consisted by global guilt, distress and guilt cognitions. Guilt cognitions can further be divided into Hindsight-Bias/Responsibility, Wrongdoing, and Lack of Justification subscales. Analyses were conducted using Pearson's correlation coefficient and analysis of covariance.

Results Results indicated ISI scores were significantly positively associated with PSS scores ( $\mathrm{r}=$ $0.620, \mathrm{p}<0.01)$ and the distress subscale of the TGRI $(\mathrm{r}=0.488, \mathrm{p}<0.01)$, and negatively associated with guilt cognitions $(r=-0.423, p<0.01) .53 .5 \%(n=23)$ of the sample met criteria for clinical insomnia using ISI cut-off scores of 15 . Participants in the insomnia group scored significantly lower in overall guilt cognitions $(\mathrm{p}<0.001)$ and significantly higher in distress $(\mathrm{p}=0.001)$ than the non-insomnia group after controlling for depression. Among the subscales of guilt cognitions, hindsight-bias/responsibility was significantly lower in the insomnia group $(\mathrm{p}<0.001)$.

Conclusions Guilt can sometimes be adaptive in trauma patients as it may work as a catalyst in cognitively processing their trauma. Our results indicate that individuals with insomnia report lower guilt cognition. This may subsequently interfere with their ability to process the traumatic experience and effectively cope with their situation.

Sleep Med Res 2018;9(1):39-45

\section{INTRODUCTION}

Posttraumatic stress disorder (PTSD) is a psychological disorder that can occur after a traumatic experience or witnessing a shocking event. According to the National Co-morbidity Survey, the lifetime prevalence of PTSD was approximately 6.8\% [1]. Patients with PTSD often experience several physical and psychological symptoms, with sleep disturbance being one of the most prominent symptoms. Sleep-related symptoms of patients with PTSD include sleep onset and maintenance difficulties and frequent nightmares as a result of traumatic events [2]. Subsequently, sleep difficulties such as insomnia can affect the onset and maintenance of PTSD symptoms [3]. Additionally, exposure to certain types of trauma such as sexual trauma, physical assault, and sudden death is associated with two- to three-fold increased 
risk of insomnia [4]. According to Steine et al. [5], individuals who were sexually abused reported more frequent sleep problems compared to non-abused individuals. Among them, the incidence of sleep disorder symptoms was especially high in rape and sexually abused victims.

The majority of theories suggest that cognitive and emotional responses play an important role in the development and maintenance of PTSD after trauma. Guilt may be especially relevant in the process of dealing with post-traumatic events [6]. Trauma-related guilt is an emotion that involves an evaluation of one's role in a negative event. In particular, guilt involves a negative assessment of a particular behavior, such as "I should have known better." A negative perception of their role after the trauma event may temporarily contribute to PTSD symptoms. However, it is less painful over time because the assessment of their role affects the process of dealing with the trauma [7]. Trauma related Guilt could be related to subsequent trauma-related sleep disturbance [8].

According to Ham et al. [9], chronic insomnia patients tend to have higher guilt-proneness. This characteristic suggests that guilt is particularly salient among those experiencing chronic insomnia. Prior research has not, however, specifically investigated the role of trauma-related guilt in PTSD patients with insomnia. In theory, traumatic events cause conflicts in the process of integrating individuals with their schema and their misbehavior. These conflicts are related to sleep disturbances [8]. Thus, the current study aimed to investigate the differences in trauma-related guilt cognitions between females of sexual violence who reported high and low symptoms of insomnia.

\section{METHODS}

\section{Participants and Procedures}

Participants were 43 females who were victims of sexual assault, ranging in age from 18 to 49 years old. All participants were recruited into the study after visiting the Sunflower Center of Southern Gyeonggi province, a trauma center specifically for females of sexual assault governed by the Korean Ministry of Gender Equality and Family. All data was collected between 2015 and 2016. The present study protocol was reviewed and approved by the Institutional Review Board of Sungshin Women's University (IRB No. SSWUIRB 2017-004). Only participants who completed written consent forms were included in the study. All participants visited the center within an average of 4 days after trauma and completed questionnaires after their initial visit.

\section{Measures}

\section{Insomnia Severity Index}

The Insomnia Severity Index (ISI) is a 7-item questionnaire that evaluates insomnia symptom severity [10]. Each of the ISI items are scored on a 5-point Likert scale ranging from 0 to 4 . A total score is calculated by summing up the whole items, ranging from 0 to 28, with higher scores indicating greater insomnia severity. According to Cho et al. [11], a cut-off score of 15 was suggested for the Korean ISI, and this study also classified insomnia groups based on this score. Internal consistency of this questionnaire was 0.90 in this sample.

\section{PTSD Symptom Scale-Self Report}

The PTSD Symptom Scale-Self Report (PSS-SR) is a 17-item self-report questionnaire that assesses PTSD symptoms, including re-experiencing, avoidance, and hyper-arousal symptoms [12]. Each of the items is rated on a 4-point Likert scale. The total PSS-SR score is the sum of all items, with higher scores indicating PTSD severity. The PSS-SR used in this study was translated as Hong and Park [13]. Internal consistency of this questionnaire was 0.93 in this sample.

\section{Trauma-Related Guilt Inventory}

The Trauma-Related Guilt Inventory (TRGI) is a 32 item questionnaire evaluating guilt associated with a particular trauma event [14]. Each of the items is measured on a 5-point Likert scale, ranging from 0 to 4 . The TRGI consists of three subscales: global guilt, distress and guilt cognitions. Guilt cognitions can further be divided into Hindsight-Bias/Responsibility (e.g., I was responsible for causing what happened), Wrongdoing (e.g., I had some thoughts or beliefs that I should not have had), and Lack of Justification (e.g., I had good reasons for doing what I did) subscales. The higher the average score of each item, the higher the perception of guilt in the trauma. In this study, we used the TRGI developed by Kubany et al. [14]. Internal consistency of this questionnaire was 0.82 in this sample.

\section{Beck Depression Inventory}

The Beck Depression Inventory is a 21-item self-report questionnaire measuring severity of depressive symptoms [15]. Each item is rated on a 4-point Likert scale of 0 to 3, with totals ranging from 0 to 63 . Higher scores reflect more depressive symptoms. In this study, we used the Korean version of the Beck Depression Scale, which was translated by Lee and Song [16]. Internal consistency of this questionnaire was 0.93 in this sample.

\section{Statistical Analyses}

Correlational analyses were conducted using Pearson's correlation coefficient between insomnia symptoms, PTSD symptom and trauma-related guilt. All participants were divided into two groups based on their ISI scores. Analysis of covariance (ANCOVA) was conducted to analyze differences in trauma-related guilt between the two groups after controlling for depression. Test of normality in the TRGI score were conducted using the Shapiro-Wilk test. Assuming normality, the AN- 
COVA would be performed. TRGI distress data is not normal, a nonparametric approach (i.e., ranked ANCOVA) would be performed. All statistical analyses were conducted using SPSS 21.0 (IBM Corp., Armonk, NY, USA).

\section{RESULTS}

\section{Demographic Information}

Demographic characteristics of the participants are shown in Table 1. All participants were females who were victims of sexual assault (mean age $26.56 \pm 7.81$ ), with $88.1 \%(n=37)$ of the sample experiencing a single traumatic event and $11.9 \%(n=5)$ experiencing multiple traumatic events. In our sample, there were 23 patients in the insomnia group and 20 patients in the non-insomnia group. The average ISI score of the insomnia group was $21.39( \pm 3.86)$ and the non-insomnia group was 11.05 $( \pm 3.38)$. Differences in PTSD symptom between the Insomnia group and non-Insomnia group can be found in Table 2. Based on the group difference test result about the severity of the insomnia, significant group difference was found in avoidance $(\mathrm{p}<$ $0.006)$, intrusion $(\mathrm{p}<0.003)$, and hyperarousal $(\mathrm{p}<0.012)$ the subfactors of PSS symptoms, respectively. However, both groups reported avoidance as the most severe symptoms compared to other symptoms.

\section{Association between Insomnia Symptom, PTSD Symptom and Trauma-Related Guilt}

ISI scores were significantly positively associated with PSS

Table 1. Demographic information $(n=43)$

\begin{tabular}{|c|c|c|c|}
\hline & $\begin{array}{l}\text { Total } \\
\text { sample } \\
\text { n (\%) }\end{array}$ & $\begin{array}{l}\text { Insomnia } \\
\text { group } \\
\mathrm{n}(\%)\end{array}$ & $\begin{array}{c}\text { Non-insomnia } \\
\text { group } \\
\mathrm{n}(\%)\end{array}$ \\
\hline \multicolumn{4}{|l|}{ Sex } \\
\hline Female & $43(100)$ & $23(100)$ & $20(100)$ \\
\hline \multicolumn{4}{|l|}{ Marital status } \\
\hline Single & $34(82.9)$ & $18(81.8)$ & $16(84.2)$ \\
\hline Married & $2(4.9)$ & $2(9.1)$ & - \\
\hline Divorced & $5(12.2)$ & $2(9.1)$ & 3 (15.8) \\
\hline Missing & 2 & 1 & 1 \\
\hline \multicolumn{4}{|l|}{ Case duration } \\
\hline Single traumatic event & $37(88.1)$ & $20(90.9)$ & $17(85.0)$ \\
\hline Multiple traumatic event & $5(11.9)$ & $2(9.1)$ & $3(15.0)$ \\
\hline Missing & 1 & 1 & - \\
\hline \multicolumn{4}{|l|}{ Report time since trauma } \\
\hline Within 24 hours & $28(65.1)$ & $16(69.6)$ & $12(60.0)$ \\
\hline Within 1 week & $12(27.9)$ & $5(21.7)$ & $7(35.0)$ \\
\hline Within 1 month & $1(2.3)$ & $1(4.3)$ & $1(5.0)$ \\
\hline More than three months & $2(4.7)$ & $1(4.3)$ & - \\
\hline
\end{tabular}

scores $(r=0.620, p<0.01)$ and the distress subscale of the TGRI $(r=0.488, p<0.01)$. ISI scores were also negatively associated with guilt cognitions $(r=-0.423, p<0.01)$. Results indicate that higher severity of insomnia was associated with more PTSD symptoms, more trauma-related distress, and less perception of trauma-related guilt (Table 3 for correlates).

\section{Trauma-Related Guilt Based on Insomnia Severity}

The insomnia group scored significantly lower in overall guilt cognitions $[\mathrm{F}(2,39)=16.760, \mathrm{p}<0.001]$ and significantly higher in distress $[\mathrm{F}(2,39)=11.599, \mathrm{p}=0.001]$ than the non-insomnia group after controlling for depression. Among the subscales of guilt cognitions, hindsight-bias/responsibility was significantly lower in the insomnia group $[F(2,39)=18.100, p<0.001]$. Results are shown in Table 4.

\section{DISCUSSION}

The current study investigated trauma-related guilt in female victims of sexual violence based on insomnia severity. While PTSD patients experience sleep disturbance and psychological changes after trauma [17], trauma-related guilt in this population is an area that has yet to be investigated in depth. In this respect, this is the first study to examine trauma-related guilt in PTSD patients with sexual trauma in relation to sleep disturbance. The main results of our study indicate that individuals with higher levels of insomnia symptoms report higher distress and lower guilt cognition after controlling for depression.

\section{Sleep Disturbance in PTSD Patients with Sexual Violence History}

In our sample, 53.7\% reported having clinical levels of insomnia, with those in the high risk group reporting particular difficulty with sleep initiation and maintenance. This is consistent with past studies that have found that individuals with sexual abuse history had longer sleep latencies and frequent nightmares than those without abuse history Steine et al. [5]. According to another previous study, $63 \%$ of female who experienced sexual assault and had PTSD complained of pain by nightmares and experienced difficulty maintaining sleep due to nightmares [18]. PTSD patients may also experience fear of sleep due to

Table 2. Difference in posttraumatic stress disorder symptoms based on insomnia $(n=43)$

\begin{tabular}{lccc}
\hline & $\begin{array}{c}\text { Insomnia group } \\
(\mathrm{n}=23)\end{array}$ & $\begin{array}{c}\text { Non-insomnia group } \\
(\mathrm{n}=20)\end{array}$ & $\mathrm{p}$ \\
\cline { 2 - 3 } & Mean rank & Mean rank & \\
\hline Intrusion & 26.48 & 15.47 & $0.003^{* *}$ \\
Avoidance & 26.17 & 15.84 & $0.006^{* *}$ \\
Hyperarousal & 25.15 & 15.69 & $0.012^{*}$ \\
\hline${ }^{*} \mathrm{p}<0.05{ }^{* *} \mathrm{p}<0.01$. & &
\end{tabular}


Table 3. Pearson's correlation coefficient between ISI and TRGI subscales $(n=43)$

\begin{tabular}{|c|c|c|c|c|c|c|c|c|c|c|c|c|c|}
\hline & Subscale & 1 & 2 & 3 & 4 & 5 & $5-1$ & $5-2$ & $5-3$ & 6 & 7 & M & $\mathrm{SD}$ \\
\hline ISI & 1. Insomnia severity & - & & & & & & & & & & 16.581 & 6.340 \\
\hline PSS & 2. PTSD symptom & $0.620^{* *}$ & - & & & & & & & & & 29.738 & 12.156 \\
\hline \multirow[t]{6}{*}{ TRGI } & 3. Global guilt & 0.007 & -0.091 & - & & & & & & & & 1.810 & 0.802 \\
\hline & 4. Distress & $0.488^{* *}$ & * $0.753^{* *}$ & -0.197 & - & & & & & & & 3.264 & 0.766 \\
\hline & 5. Guilt cognitions & $-0.423^{* *}$ & * -0.148 & $-0.376^{*}$ & 0.005 & - & & & & & & 2.176 & 0.835 \\
\hline & 5-1. Hindsight-bias/responsibility & $-0.330^{*}$ & -0.094 & $-0.420 * *$ & 0.005 & $0.883^{* *}$ & - & & & & & 2.258 & 0.946 \\
\hline & 5-2. Wrongdoing & -0.085 & 0.215 & -0.191 & 0.275 & $0.635^{* *}$ & $0.452^{* *}$ & - & & & & 2.231 & 0.962 \\
\hline & 5-3. Lack of justification & $-0.394^{* *}$ & $*-0.230$ & -0.085 & -0.124 & $0.640^{* *}$ & $0.354^{*}$ & 0.296 & - & & & 2.343 & 0.918 \\
\hline \multirow[t]{2}{*}{ BDI } & 6. Depression & 0.228 & $0.638^{* *}$ & $-0.438^{* *}$ & $0.667^{* *}$ & $0.352 *$ & $0.392^{*}$ & $0.472^{* *}$ & 0.024 & - & & 32.814 & 12.487 \\
\hline & $\begin{array}{l}\text { 7. Depression } \\
\text { (except sleep related item) }\end{array}$ & 0.218 & $0.652^{* *}$ & $-0.427^{* *}$ & $0.671^{* *}$ & $0.358^{*}$ & $0.394^{* *}$ & $0.481^{* *}$ & 0.036 & $0.997^{* *}$ & - & 31.000 & 11.982 \\
\hline
\end{tabular}

${ }^{*} \mathrm{p}<0.05 .{ }^{* *} \mathrm{p}<0.01$.

ISI: Insomnia Severity Index, PSS: PTSD Symptom Scale, PTSD: posttraumatic stress disorder, TRGI: Trauma-Related Guilt Inventory, BDI: Beck Depression Inventory.

Table 4. Differences of trauma-related guilt between insomnia group and non-insomnia groups $(n=43)$

\begin{tabular}{|c|c|c|c|c|c|c|c|}
\hline & \multicolumn{2}{|c|}{ Non-insomnia $(\mathrm{n}=20)$} & \multicolumn{2}{|c|}{ Insomnia $(\mathrm{n}=23)$} & \multirow{2}{*}{$\mathrm{F}$} & \multirow{2}{*}{$\mathrm{p}$} & \multirow{2}{*}{$\eta^{2}$} \\
\hline & $\mathrm{M}$ & $\mathrm{SD}$ & $\mathrm{M}$ & $\mathrm{SD}$ & & & \\
\hline \multicolumn{8}{|l|}{ Trauma-related guilt scales } \\
\hline Global guilt & 1.75 & 0.88 & 1.86 & 0.75 & 0.906 & 0.347 & 0.023 \\
\hline Distress & 2.93 & 0.84 & 3.55 & 0.57 & 11.599 & $0.001^{* *}$ & - \\
\hline Guilt cognitions & 2.59 & 0.67 & 1.82 & 0.81 & 16.760 & $0.000^{* * *}$ & 0.295 \\
\hline \multicolumn{8}{|l|}{ Guilt cognitions subscales } \\
\hline Hindsight-bias/responsibility & 2.73 & 0.79 & 1.85 & 0.90 & 18.100 & $0.000^{* * *}$ & 0.312 \\
\hline Wrongdoing & 2.37 & 0.76 & 2.11 & 1.11 & 2.059 & 0.159 & 0.049 \\
\hline Lack of justification & 2.52 & 0.78 & 2.19 & 1.01 & 1.447 & 0.236 & 0.035 \\
\hline
\end{tabular}

Covariates: Beck Depression Inventory scores (depression).

${ }^{* *} \mathrm{p}<0.05{ }^{* * *} \mathrm{p}<0.01$.

SD: standard deviation.

trauma-related nightmares or dreams, thereby experiencing difficulty with sleep initiation and maintenance [19]. Additionally, for people with sexual violence history, the bedroom may be perceived as threatening because sexual abuse may have occurred in the bedroom, thus making it a salient stimulus. This can lead the patient to perceive additional threats to safety and protection, which may subsequently exacerbate sleep disturbance [20].

PTSD patients experiencing sleep disorders can also be explained from a biological perspective. Individuals who have experienced a shocking event have fight-or-flight response to protect themselves. However, even after the actual risk has disappeared, the fight-or-flight response may remain and cause overactivation of the sympathetic nervous system, resulting in heightened arousal level. In addition to an overactive autonomic nervous system, the hypothalamus-pituitary-adrenal axis may be activated when the patient has been exposed to multiple traumas. This may lead to frequent awakenings that interfere with normal sleeping, and attenuates the recovery effect of sleep, resulting in insomnia [21]. Thus, females with sexual trauma may be more vulnerable to insomnia than PTSD patients with other types of trauma.

\section{Lower Guilt Cognition Associated with Higher Insomnia Risk}

In our study, participants who were at higher risk for insomnia reported higher distress and lower guilt cognition compared to individuals who had lower insomnia risk. This result supports the findings that sleep in PTSD patients of sexual violence is associated with trauma-related cognitive and emotional factors $[17,22]$. Individuals exposed to an unexpectedly shocking event require processing to cope with the event. Individuals exposed to trauma experience inconsistencies in existing memory structures due to negative emotional information related to traumatic experiences. One way trauma victims try to integrate the new traumatic experience with existing cognitive systems 
is by recalling traumatic events over time. During this process, the failure to integrate trauma-related information causes symptoms of PTSD [23]. Sleep in PTSD patients plays an important role in the integration and processing of traumatic events [24]. In particular, sleep is associated with the regulation of cognitive and emotional brain processes, and abnormalities in sleep function are reported to be difficulties in cognitive and emotional processing $[25,26]$. Importantly, high levels of insomnia symptoms were associated with low guilt cognition. This result speculates that low guilt cognition may reflect active avoidance of processing trauma-related cues in this population. This was also supported in our study, as the insomnia group reported highest on avoidance compared to other PTSD symptoms.

The results of having low guilt cognition in insomnia patients are somewhat inconsistent with previous studies. This may be reflective of previous studies investigating the role of guilt without differentiating it from shame, which is a similar emotion. In previous studies, most studies have reported a positive association between guilt and PTSD symptoms. But in some studies, when controlling for the effects of shame, PTSD symptom and guilt were found to be negatively associated [27-29]. Shame is frequently activated simultaneously with guilt after trauma, and both shame and guilt are similar in that they are associated with evaluating feelings related to the self. However, in contrast to guilt, shame involves an assessment of the overall self, such as "I am a bad person" [28,30]. As in previous studies, our results suggest that guilt can serve as a protective factor for individuals in PTSD development [30-32]. In particular, it is noteworthy that the absence of experience of trauma-related guilt plays an important role in trauma-related sleep disorders. According to a study by van Minnen et al. [33], individuals with little or no guilt had no statistical room for improvement in PTSD symptoms, and individuals who actually have extreme initial scores tend to be maintained or increased after treatment. In other words, these results suggest that it may be more adaptive to face and accept negative cognitions and emotions such as guilt that are related to the trauma experience. Especially, focusing on the perception of dysfunctional behavior will provide an opportunity to search for adaptive negative emotions.

In our study, we studied trauma-related guilt, which was distinguished from shame and the sub-factors of guilt was examined in detail. Our results reported significantly lower guilt in the insomnia group. Especially, it showed significantly low score in 'Hindsight-Bias/Responsibility' in individuals with insomnia. Hindsight-Bias/Responsibility is a guilt-related cognition that reflects how much an individual believes they possess knowledge of the outcome of an event and predict its outcome. For example, a sexual assault victim with high hindsight bias/responsibility would believe that they should have "known something goes wrong" or "directed to the other direction," and they feel responsible for not revealing the past abuse. This may lead to excessive self-blame by believing that trauma survivors were overburdened by trauma events or related negative consequences and were able to avoid trauma events [7,34]. If a trauma survivor falsely believes he or she had pre-outcome knowledge that would have enabled him or her to change the course of trauma events, it follows logically that they "chose" not to prevent the event and thereby is responsible to some extent for having caused the event [7]. According to Ianoff-Bulman [35], it has been proposed that the protective factor can be a function of the type of self-blame. Self-blame for specific behaviors that change the cause of traumatic experiences into controllable or modifiable aspects such as specific behaviors can be more adaptive compared to characterological self-blame attributed to uncontrollable aspects such as one's personality. In a previous study of self-blame, behavioral self-blame in trauma events showed that event-related pain decreased over time [36], and in a study of victims of sexual assault, a group with high self-criticism behavior was found to be adaptive in coping with trauma events [37]. More specifically, the post-confirmation bias is high when you agree with the question "I could have prevented what happened," "I blame myself for something I did, thought, or felt." Higher scores on these questions reflect more negative the behavior that failed, which may facilitate an active process in restructuring the existing cognitive system and subsequently help recovery. According to some studies, higher guilt tendency after the negative experience was more closely associated with the degree that a respondent accepts his or her experience as it is [29]. Considering this point, sleep can be an important factor in dealing with traumatic events. Especially, when females who experience sexual violence display lower guilt, it may lead to avoidance of evaluating and exploring the cause of the event and moving past blaming themselves for controllable or modifiable aspects of the traumatic event.

\section{Clinical Implications}

The relationship between insomnia and trauma-related guilt in females with sexual violence has several important clinical implications. Previous studies have shown that PTSD patients with insomnia have worse clinical outcomes than patients who have PTSD without insomnia. In addition, patients with previous sexual violence experiences reported significantly more than those who experienced other trauma. From a clinical point of view, our results suggest that female with a history of sexual violence may be more vulnerable to insomnia and that access to sleep problems is important in treating them. Previous studies have shown that patients with PTSD frequently have negative mood states, such as depression, and that the effects of these moods can have a significant impact on PTSD-related symptoms $[38,39]$. This study is meaningful in that it explains the relationship between two concepts independent of the control of depression by statistical methods. Many PTSD psychotherapy studies have shown that facing and accepting emotions associated with trauma events was effective in alleviating PTSD 
symptoms $[33,40]$. Trauma-related guilt is a form of emotion that includes self-assessment of traumatic experiences and is often mixed with shame. Our results suggest that assessing traumatic guilt in the context of sleep disturbance in PTSD patients, especially females with sexual trauma, may be helpful in the treatment of women who experience sexual abuse. If the trauma is perceived to be a violation of a specific rule, it will be a strategy to reduce the individual suffering from trauma, leading to a process of activating a fundamental belief in oneself or confirming their experience. In this respect, future studies should focus on whether providing sleep specific treatment in addition to traditional psychotherapy may help improve sleep, and subsequently affect trauma-related guilt and activate the recovery process.

\section{Limitations}

The limitations of this study and suggestions for future research are as follows. First, this study had a small sample size. Additional studies with larger sample sizes are needed to further elucidate the role of sleep in the process of coping with trauma. Second, the unity of the initial assessment period after the incident was insufficient. Most of the initial assessments were made within average of 6 days after their initial visit, but the unity of the period from one month to three months after the occurrence of the incident was insufficient. Therefore, future studies need to consider the evaluation period after the incident. Third, in this study, TRGI was used to measure Trauma-Related Guilt. TRGI includes perceived wrong-doing, responsibility and self-blame that may be particularly relevant to the cognitive, affective, and behavioral components of guilt. This tool is reported to be an excellent measure of the guilt properties, but it is difficult to completely exclude the possibility of also measuring shame. In future studies, it is necessary to distinguish between guilt and shame, including measures of shame. Also, because this study is a retrospective study, it did not include a questionnaire to evaluate the coping strategy or post-traumatic perception in advance. In future studies, it is necessary to verify the cognitive factors after trauma, whether using low avoidance coping strategies or low guilt feelings. Fourth, the present study has limitations in revealing the causal relationship in the nature of the study because it was a cross-sectional study. Therefore, it will be necessary in future studies to investigate the path of insomnia symptoms and trauma-related guilt level of women who have experienced sexual violence by supplementing these limitations. Finally, although this study suggests that guilt may serve as a protective factor, there are limits to understanding the impact of long-term trauma-related guilt. Future research should investigate the longitudinal effects of guilt on the recovery of PTSD symptoms.

\section{Conclusion}

Results from the current study indicated the association between insomnia and trauma-related guilt in females with sexu- al violence history. Our findings suggest that sleep disturbance in these individuals may be associated with lower guilt, which may prevent these individuals from actively processing their trauma experience. These results imply that it will be important for clinicians to assess and be mindful of trauma-related guilt, especially differentiated from shame. Furthermore, future studies should focus on the role of guilt and develop and incorporate specific interventions that can actively process guilt in this population.

\section{Conflicts of Interest}

The authors have no financial conflicts of interest.

\section{Authors' Contribution}

Study design: Han SR, Chang HY, Suh SY. Data collection: Chung YK, Kim MR, Lee HB, Kim YJ, Chang HY. Data analysis: Han SR. Interpretation of results: Han SR, Chang HY, Suh SY. Preparation of the manuscript: Han SR, Lee HB, Kim YJ, Chang HY, Suh SY. Critical review of the manuscript: Chung YK, Kim MR. Writing-original draft: Han SR. Writingreview \& editing: Chang HY, Suh SY.

\section{REFERENCES}

1. Kessler RC, Berglund P, Demler O, Jin R, Merikangas KR, Walters EE. Lifetime prevalence and age-of-onset distributions of DSM-IV disorders in the National Comorbidity Survey Replication. Arch Gen Psychiatry 2005;62:593-602.

2. Harvey AG, Jones C, Schmidt DA. Sleep and posttraumatic stress disorder: a review. Clin Psychol Rev 2003;23:377-407.

3. Cox R, McIntyre W, Olatunji B. Interactive effects of insomnia symptoms and trauma exposure on PTSD: examination of symptom specificity. Psychol Trauma 2017 Nov 20 [Epub ahead of print]. https://doi. org/10.1037/tra0000336.

4. Brown TSH, Akeeb A, Mellman TA. The role of trauma type in the risk for insomnia. J Clin Sleep Med 2015;11:735-9.

5. Steine IM, Krystal JH, Nordhus IH, Bjorvatn B, Harvey AG, Eid J, et al. Insomnia, nightmare frequency, and nightmare distress in victims of sexual abuse: the role of perceived social support and abuse characteristics. J Interpers Violence 2012;27:1827-43.

6. Pugh LR, Taylor PJ, Berry K. The role of guilt in the development of post-traumatic stress disorder: a systematic review. J Affect Disord 2015; 182:138-50.

7. Kubany ES, Manke FP. Cognitive therapy for trauma-related guilt: conceptual bases and treatment outlines. Cognitive and Behavioral Practice 1995;2:27-61.

8. Dedert EA, Dennis PA, Cunningham KC, Ulmer CS, Calhoun PS, Kimbrel N, et al. Roles of guilt cognitions in trauma-related sleep disturbance in military veterans with posttraumatic stress disorder. Behav Sleep Med 2018 Feb 26 [Epub ahead of print]. https://doi.org/10.1080/ 15402002.2018.1435544.

9. Ham BJ, Kim L, Suh K-Y. Personality characteristics of chronic insomniacs. J Korean Neuropsychiatr Assoc 1998;37:234-42.

10. Bastien CH, Vallières A, Morin CM. Validation of the insomnia severity index as anoutcome measure for insomnia research. Sleep Med 2001; 2:297-307.

11. Cho YW, Song ML, Morin CM. Validation of a korean version of the insomnia severity index. J Clin Neurol 2014;10:210-5.

12. Foa EB, Riggs DS, Dancu CV, Rothbaum BO. Reliability and validity of a brief instrument for assessing post-traumatic stress disorder. $J$ Traumatic Stress 1993;6:459-73.

13. Hong C-H, Park Y-S. The effects of violence of husband on psychopathology of wife. Res Psychol 2001;2:1-20. 
14. Kubany ES, Haynes SN, Abueg FR, Manke FP, Brennan JM, Stahura C. Development and validation of the trauma-related guilt inventory (TRGI). Psychol Assess 1996;8:428-44.

15. Beck AT, Steer RA, Brown GK. Beck depression inventory-II. San Antonio 1996;78:490-8.

16. Lee YH, Song JY. A study of the reliability and the validity of the BDI, SDS, and MMPI-D Scales. Korean J Clin Psychol 1991;10:98-113.

17. Germain A. Sleep disturbances as the hallmark of PTSD: where are we now? Am J Psychiatry 2013;170:372-82.

18. Krakow B, Tandberg D, Barey M, Scriggins L. Nightmares and sleep disturbance in sexually assaulted women. Dreaming 1995;5:199-206.

19. Neylan TC, Marmar CR, Metzler TJ, Weiss DS, Zatzick DF, Delucchi $\mathrm{KL}$, et al. Sleep disturbances in the Vietnam generation: findings from a nationally representative sample of male Vietnam veterans. Am J Psychiatry 1998;155:929-33.

20. Noll JG, Trickett PK, Susman EJ, Putnam FW. Sleep disturbances and childhood sexual abuse. J Pediatr Psychol 2006;31:469-80.

21. Sherin JE, Nemeroff CB. Post-traumatic stress disorder: the neurobiological impact of psychological trauma. Dialogues Clin Neurosci 2011; 13:263-78.

22. Foa EB, Huppert JD, Cahill SP. Emotional processing theory: an update. In: Rothbaum BO. Pathological anxiety: Emotional processing in etiology and treatment. New York: Guilford Press 2006;3-24.

23. Brewin CR, Holmes EA. Psychological theories of posttraumatic stress disorder. Clin Psychol Rev 2003;23:339-76.

24. Brewin CR, Dalgleish T, Joseph S. A dual representation theory of posttraumatic stress disorder. Psychol Rev 1996;103:670-86.

25. Walker MP. The role of sleep in cognition and emotion. Ann N Y Acad Sci 2009;1156:168-97.

26. Kahn M, Sheppes G, Sadeh A. Sleep and emotions: bidirectional links and underlying mechanisms. Int J Psychophysiol 2013;89:218-28.

27. Leskela J, Dieperink M, Thuras P. Shame and posttraumatic stress dis- order. J Trauma Stress 2002;15:223-6.

28. Tangney JP, Stuewig J, Mashek DJ. Moral emotions and moral behavior. Annu Rev Psychol 2007;58:345-72.

29. Song SM. A comparative study between shame-proneness and guiltproneness by psychological traits. Korean J Counsel Psychotherapy 2008; 20:125-44.

30. Lewis HB. Shame and guilt in neurosis. Psychoanal Rev 1971;58:419.

31. Tangney JP, Stuewig J, Mashek DJ. Moral emotions and moral behavior. Annu Rev Psychol 2007;58:345-72.

32. Kim S, Thibodeau R, Jorgensen RS. Shame, guilt, and depressive symptoms: a meta-analytic review. Psychol Bull 2011;137:68-96.

33. van Minnen A, Zoellner LA, Harned MS, Mills K. Changes in comorbid conditions after prolonged exposure for PTSD: a literature review. Curr Psychiatry Rep 2015;17:549.

34. Fischhoff B. Hindsight is not equal to foresight: the effect of outcome knowledge on judgment under uncertainty. J Experim Psychol: Hum Percept Perform 1975;1:288-99.

35. Ianoff-Bulman R. Shattered assumptions. 1st ed. New York: Free Press 1992.

36. Delahanty DL, Herberman HB, Craig KJ, Hayward MC, Fullerton CS, Ursano RJ, et al. Acute and chronic distress and posttraumatic stress disorder as a function of responsibility for serious motor vehicle accidents. J Consult Clin Psychol 1997;65:560-7.

37. Koss MP, Figueredo AJ, Prince RJ. Cognitive mediation of rape's mental, physical, and social health impact: tests of four models in crosssectional data. J Consult Clin Psychol 2002;70:926-41.

38. Clark LA, Watson D. Constructing validity: basic issues in objective scale development. Psychol Assess 1995;7:309-19.

39. Wilson JP, Drozdek B, Turkovic S. Posttraumatic shame and guilt. Trauma Violence Abuse 2006;7:122-41.

40. Wenzlaff RM, Wegner DM. Thought suppression. Annu Rev Psychol 2000;51:59-91. 